\title{
Entwurf robuster Regelungen
}

Von Dr.-Ing. Kai Müller

Akademischer Rat am Institut

für Regelungstechnik der

Technischen Universität Braunschweig

Mit 97 Bildern und 2 Tabellen

돈 Springer Fachmedien Wiesbaden GmbH 1996 
Die Deutsche Bibliothek - CIP-Einheitsaufnahme

Müller, Kai:

Entwurf robuster Regelungen : mit 2 Tabellen / von Kai Müller.

ISBN 978-3-519-06173-1

ISBN 978-3-663-12091-9 (eBook)

DOI 10.1007/978-3-663-12091-9

Das Werk einschließlich aller seiner Teile ist urheberrechtlich geschützt. Jede Verwertung außerhalb der engen Grenzen des Urheberrechtsgesetzes ist ohne Zustimmung des Verlages unzulässig und strafbar. Das gilt besonders für Vervielfältigungen, Übersetzungen, Mikroverfilmungen und die Einspeicherung und Verarbeitung in elektronischen Systemen.

(C) Springer Fachmedien Wiesbaden 1996

Ursprünglich erschienen bei B.G. Teubner Stuttgart 1996 


\section{Vorwort}

In Abständen von etwa 20 Jahren konnten in der Regelungstechnik grundsätzliche Neuerungen beobachtet werden. Nachdem in den 40er Jahren eine systematische Behandlung von Regelkreisen im Frequenzbereich entwickelt wurde (Bode, Nyquist, Ziegler und Nichols, Wiener), verlagerte sich um 1960 das Interesse der Theoretiker auf den Zeitbereich, den Zustandsraum und optimale Regelung (Luenberger, Kalman). Die Verfahren basieren auf Prozeßmodellen, und es stellte sich heraus, daß häufig in der praktischen Anwendung Probleme aufgrund von Modell- oder Parameterunsicherheiten auftreten. Dies motivierte die Entwicklung der robusten Regelungen in den 80er Jahren, die explizit bei Anwesenheit von Unsicherheiten Stabilität bzw. die Einhaltung bestimmter Qualitätsmerkmale gewährleisten ([16], [17], [68]) und somit große praktische Relevanz aufweisen.

In diesem Buch sollen Theorie und Einsatz der in den 80er Jahren entwickelten Norm-optimalen Regelungen vermittelt werden. Selbstverständlich handelt es sich hierbei nur um eine Facette der zahlreichen neuen Entwicklungen auf dem Gebiet der Entwurfs- und Analyseverfahren. Insbesondere die $\mathrm{H}_{\infty}$-optimalen Regler und die $\mu$-Synthese haben jedoch in den letzten 10 Jahren entscheidend dazu beigetragen, daß sich das Bild der "modernen" Regelungstechnik merklich gewandelt hat.

Obwohl die Norm-optimalen Regelungen einen neuen Ansatz darstellen, so ist doch die Entwicklung ohne die Ergebnisse früherer Verfahren undenkbar. Aus didaktischen Gründen werden deshalb den robusten Regelungen Verfahren wie Polvorgabe, quadratisch optimale Regelung oder LQG vorangestellt.

In Kapitel 7 schließt sich eine Einführung in Normen für Signale und Systeme an. Die Entwicklung der koprimen Faktorisierung und der Reglerparametrierung (Kapitel 8) ist sowohl ein eigenständiges, universelles Entwurfsverfahren als auch die historische Grundlage für die 2- und $\infty$-Norm-optimalen Regelungen (Kapitel 9, 10 und 11). Die Theorie wird vollständig am Beispiel von Eingrößenstrecken hergeleitet. Im 12. Kapitel erfolgt die Verallgemeinerung auf Mehrgrößensysteme im Zustandsraum. Ein Mehrgrößenproblem entsteht auch bei Eingrößenstrecken, wenn mehrere Anforderungen zu berücksichtigen sind. 
Es wurden konsequent die in der internationalen Literatur üblichen Bezeichnungen verwendet. Dabei wurde bewußt in Kauf genommen, daß Symbole in verschiedenen Kapiteln unterschiedliche Bedeutung tragen können. So hat beispielsweise Q in Kapitel 5 über optimale Regelungen die Bedeutung einer Gewichtsmatrix, während in Kapitel 8 über koprime Faktorisierung Q(s) die Übertragungsfunktion für die Regler-Parametrierung ist.

Die bisherigen Ergebnisse werden im letzten Kapitel verwendet, um $\infty$-Norm-optimale Regler für unstrukturierte Unsicherheiten zu entwerfen. Mit der Einführung des strukturierten singulären Wertes $\mu$ wird gezeigt, wie Parameterunsicherheiten berücksichtigt werden können und sich ein systematischer Reglerentwurf durchführen läßt, der Regelqualität bei Anwesenheit von Unsicherheiten gewährleistet.

Auch wenn für viele Anwendungen der Aufwand für den Entwurf robuster Regelungen zu hoch erscheint, so gibt der Umgang mit diesen Verfahren doch darüber Aufschluß, welche Ergebnisse mit modernen Verfahren erreichbar sind.

Mein Dank gilt Marcus Brand und Kai Michels von der Technischen Universität Braunschweig sowie Dr. John Chiasson von der University of Pittsburgh für fachliche und menschliche Unterstützung. 


\section{Inhalt}

1 Regelungstechnische Grundlagen ............ 1

1.1 Die Begriffe der Regelungstechnik nach DIN 19226 und

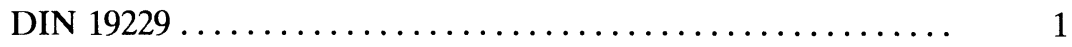

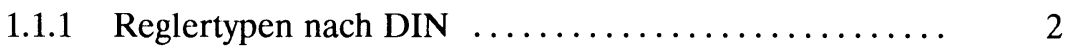

1.2 In der Regelungstheorie gebräuchliche Bezeichnungen ...... 6

1.3 Ein- und Mehrgrößensysteme $\ldots \ldots \ldots \ldots \ldots \ldots \ldots \ldots \ldots$

1.4 Grundlegende Beziehungen des geschlossenen Kreises ...... 8

1.5 Berücksichtigung von Modellunsicherheiten ............ 11

1.6 Motivierende Einführung: Die Youla-Parametrierung für stabile Strecken ............................ 15

1.6.1 Beispiel für den Eingrößenfall .............. 17

1.7 Übungsbeispiel: Youla-Parametrierung $\ldots \ldots \ldots \ldots \ldots \ldots \ldots$

1.7.1 Lösung $\ldots \ldots \ldots \ldots \ldots \ldots \ldots \ldots \ldots \ldots \ldots \ldots \ldots \ldots \ldots \ldots \ldots \ldots \ldots \ldots \ldots$

2 Übertragungsfunktion und Zustandsdarstellung . . . . . 21

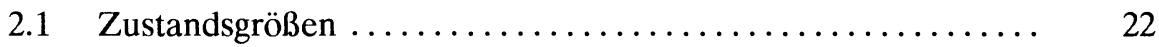

2.1.1 Regelungs-Normalform ................. 22

2.1.2 Zustandsgleichung und Ausgangsgleichung ........ 25

2.1.3 Physikalisches Beispiel: Schwingkreis ........... 26

2.2 Laplace-Transformation der Zustandsgleichungen ........ 27

2.2.1 Berechnung einer Übertragungsfunktion aus der Zustandsdarstellung ................... 29

2.3 Basistransformation im Zustandsraum ............. 30

2.4 Beobachter-Normalform ...................... 31

2.5 Übungsbeispiel: Zustandsdarstellung einer mechanischen

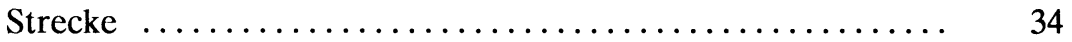




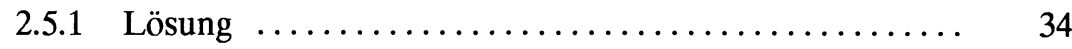

3 Zustandsregelung $\ldots \ldots \ldots \ldots \ldots \ldots \ldots \ldots \ldots \ldots \ldots, 38$

3.1 Polvorgabe mit einem Zustandsregler ................ 39

3.2 Struktur von (sI - A) $)^{-1}$, Leverrier-Algorithmus ........... 40

3.3 Berechnung des Polvorgabe-Reglers (Zustandsregelung) ...... 41

3.3.1 Beispiel für ein nicht steuerbares System .......... 45

3.4 Polvorgabe bei Regelungs-Normalform .............. 47

3.5 Zustandsregler für Führungsverhalten $\ldots \ldots \ldots \ldots \ldots \ldots \ldots . \quad 48$

3.5.1 Zustandsrealisierung des geschlossenen Kreises $\ldots \ldots . \quad 50$

3.6 Übungsbeispiel: Polvorgabe $\ldots \ldots \ldots \ldots \ldots \ldots \ldots \ldots \ldots \ldots . \quad 51$

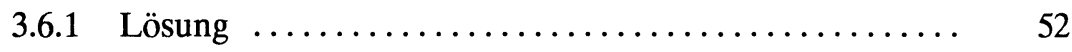

3.7 CAD-Übung: Zustandsreglerentwurf (MATLAB-Software erforderlich) .................. 55

3.7.1 MATLAB M-Files $\ldots \ldots \ldots \ldots \ldots \ldots \ldots \ldots \ldots \ldots \ldots \ldots \ldots$

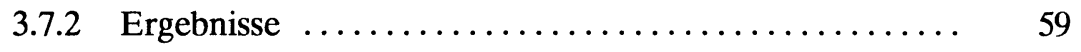

4 Beobachter $\ldots \ldots \ldots \ldots \ldots \ldots \ldots \ldots \ldots \ldots \ldots \ldots \ldots .64$

4.1 Der Entwurf des Luenberger-Beobachters $\ldots \ldots \ldots \ldots \ldots \ldots \quad 66$

4.2 Zustandsregelung mit Beobachter $\ldots \ldots \ldots \ldots \ldots \ldots \ldots \ldots .68$

4.2.1 Bestimmung des Reglers ................... 70

4.3 Übungsbeispiel: Zustandsregelung mit Beobachter ........ 71

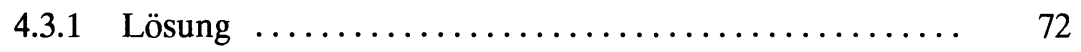

5 Optimale Zustandsregelung $\ldots \ldots \ldots \ldots \ldots \ldots \ldots \ldots . \quad 75$

$5.1 \quad$ Berechnung der optimalen Stellgröße $u(t) \ldots \ldots \ldots \ldots \ldots \ldots \quad 77$

5.2 Zusammenfassung der Lösung $\ldots \ldots \ldots \ldots \ldots \ldots \ldots \ldots \ldots \ldots \ldots \ldots \ldots$

5.3 Beispiel für eine optimale Zustandsregelung $\ldots \ldots \ldots \ldots \ldots . \quad 82$

5.4 Bewertung der Ausgangsgröße y anstelle der

Zustandsgröße x ............................ 83 
5.5 Übungsbeispiel: LQR (Linear Quadratic Regulator) . . . . . . . 84

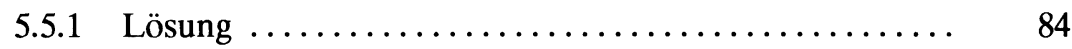

6 Kalman-Filter und LQG ..................... 88

6.1 Berechnung des Kalman-Filters $\ldots \ldots \ldots \ldots \ldots \ldots \ldots \ldots . \quad 90$

6.2 LQG (Linear Quadratic Gaussian) ............... 92

6.2.1 Berechnung des Reglers nach dem LQG-Verfahren ... 93

6.3 CAD-Übung: Kalman-Filter

(MATLAB-Software erforderlich) $\ldots \ldots \ldots \ldots \ldots \ldots \ldots \ldots .95$

6.3 .1 MATLAB M-Files ..................... 96

6.3.2 Ergebnisse $\ldots \ldots \ldots \ldots \ldots \ldots \ldots \ldots \ldots \ldots \ldots \ldots \ldots$

$7 \quad$ Normen für Signale und Systeme $\ldots \ldots \ldots \ldots \ldots \ldots \ldots \ldots$

7.1 Eigenschaften von Normen $\ldots \ldots \ldots \ldots \ldots \ldots \ldots \ldots \ldots \ldots$

7.2 Normen für Signale $\ldots \ldots \ldots \ldots \ldots \ldots \ldots \ldots \ldots \ldots \ldots \ldots \ldots$

7.2.1 Endlichkeit von Normen für Signale $\ldots \ldots \ldots \ldots \ldots \ldots$

7.3 Normen für Systeme ...................... 105

7.3.1 Endlichkeit von Normen für Systeme $\ldots \ldots \ldots \ldots \ldots . \ldots 107$

7.3.2 Berechnung der 2-Norm ............... 108

7.3.3 Berechnung der $\infty$-Norm $\ldots \ldots \ldots \ldots \ldots \ldots \ldots \ldots \ldots$

7.4 Eingangs-/Ausgangsbeziehungen ................. 110

7.5 Übungsbeispiel: Berechnung der Normen von Systemen ..... 116

7.5 .1 Lösung $\ldots \ldots \ldots \ldots \ldots \ldots \ldots \ldots \ldots \ldots \ldots \ldots \ldots \ldots \ldots \ldots \ldots \ldots \ldots \ldots$

8 Koprime Faktorisierung . . . . . . . . . . . . . . . . . 119

8.1 Berechnung von $\mathrm{X}(\mathrm{s})$ und $\mathrm{Y}(\mathrm{s})$ mit dem Algorithmus von Euklid ......................... 120

8.2 Beispiel: koprime Faktorisierung einer instabilen Strecke .... 122

8.3 Q-Parametrierung: der allgemeine Fall .............. 124

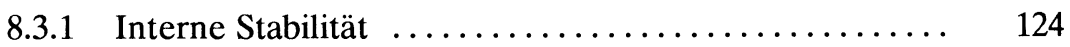


8.4 Die Menge aller $G$ stabilisierenden Regler $\ldots \ldots \ldots \ldots \ldots \ldots$

8.4.1 Herleitung der internen Stabilität für den geschlossenen Kreis mit dem parametrierten Regler ....

8.5 Entwurf eines stabilisierenden Reglers mit vorgebbaren

Eigenschaften durch koprime Faktorisierung .......... 128

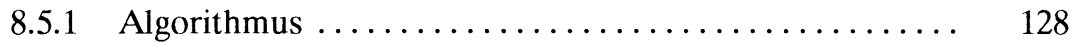

8.6 Übungsbeispiel: Reglerentwurf für eine instabile Strecke durch koprime Faktorisierung $\ldots \ldots \ldots \ldots \ldots \ldots \ldots \ldots \ldots$

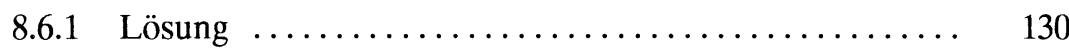

9 Modellabgleich $\ldots \ldots \ldots \ldots \ldots \ldots \ldots \ldots \ldots \ldots \ldots \ldots, 134$

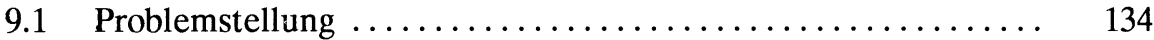

9.1.1 Beispiel für minimale $\infty$-Norm bei einer Nullstelle von $\mathrm{U}(\mathrm{s})$ in der rechten Halbebene...$\ldots \ldots \ldots \ldots \ldots$

10 Minimierung der 2 -Norm $\ldots \ldots \ldots \ldots \ldots \ldots \ldots \ldots \ldots 138$

10.1 Definition der Mengen $\varphi_{0}$ und $\varphi_{0}^{\perp} \ldots \ldots \ldots \ldots \ldots \ldots \ldots \ldots$

10.2 Berechnung des 2-Norm-optimalen $\mathrm{Q}(\mathrm{s}) \ldots \ldots \ldots \ldots \ldots \ldots$

10.3 Beispiel für eine 2-Norm-optimale Regelung ............ 142

10.4 Übungsbeispiel: 2-Norm-optimale Regelung für eine instabile Strecke ......................... 145

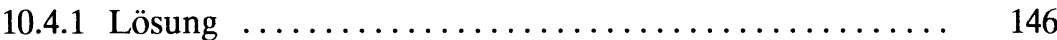

11 Minimierung der $\infty$-Norm $\ldots \ldots \ldots \ldots \ldots \ldots \ldots \ldots \ldots .149$

11.1 Nevannlinna-Pick-Interpolation ................... 149

11.1.1 NP für ein Wertepaar $a_{1}, b_{1} \ldots \ldots \ldots \ldots \ldots \ldots \ldots . \ldots . \ldots$

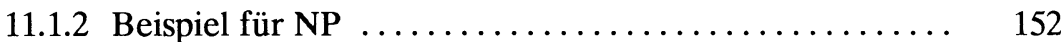

11.1.3 NP für $n$ Punkte $\ldots \ldots \ldots \ldots \ldots \ldots \ldots \ldots \ldots \ldots \ldots \ldots$

11.1.4 Beispiel: NP für 2 Wertepaare $\ldots \ldots \ldots \ldots \ldots \ldots \ldots . \ldots \ldots$

11.2 Lösung des Modellabgleich-Problems für $\|\mathrm{T}-\mathrm{UQ}\|_{\infty}$ durch NP .................................. 155 
11.2.1 Algorithmus zur Berechnung $\mathrm{Q}(\mathrm{s})$ für min $\|\mathrm{T}-\mathrm{UQ}\|_{\infty}$.

11.3 Übungsbeispiel: $\infty$-Norm-optimale Regelung einer instabilen Strecke

11.3.1 Lösung gemäß Кар. 11.2.1 ................. 159

12 Berechnung 2- und $\infty$-Norm-optimaler Regler im Zustandsraum (H2-/Ho-optimale Regler) . ......... 163

12.1 Berechnung der 2-Norm von Mehrgrößenstrecken ......... 165

12.2 Berechnung der $\infty$-Norm von Mehrgrößenstrecken ......... 167

12.3 Berechnung Norm-optimaler Regler im Zustandsraum . . . . . . 170

12.3.1 Beispiel: $\mathrm{PT}_{1}-$ Strecke mit proportionalen

Gewichtsfunktionen $\mathrm{W}_{1}$ und $\mathrm{W}_{2} \ldots \ldots \ldots \ldots \ldots \ldots \ldots$

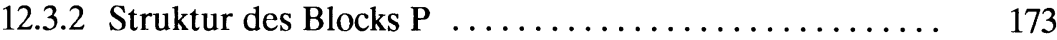

12.4 Berechnung des 2-Norm-optimalen Reglers .............. 174

12.4.1 Beispiel für eine 2-Norm-optimale Regelung mit dynamischer Stellgrößenbeschränkung ......... 175

12.5 Bedingungen für die Existenz von Lösungen für den

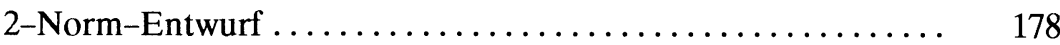

12.6 Berechnung des optimalen Reglers ................. 179

12.7 Beispiel für eine $\infty$-Norm-optimale Regelung $\ldots \ldots \ldots \ldots \ldots 181$

12.8 Bedingungen für die Existenz von Lösungen für den $\infty$-Norm-Entwurf

13 Reglerentwurf für Prozesse mit unsicheren Parametern und unstrukturierten Modellunsicherheiten

13.1 Reglerentwurf für Prozesse mit unstrukturierter

Modellunsicherheit

13.1.1 Beispiel: Entwurf eines Reglers für robuste Stabilität . . 190

13.2 Der strukturierte singuläre Wert $\mu$ (SSV) ............. 192

13.2.1 Definition von $\mu \ldots \ldots \ldots \ldots \ldots \ldots \ldots \ldots \ldots \ldots \ldots \ldots$

13.2.2 Berechnung von $\mu \ldots \ldots \ldots \ldots \ldots \ldots \ldots \ldots \ldots . \ldots . \ldots . \ldots$ 
13.3 Analyse der robusten Stabilität bei strukturierter Modellunsicherheit ............................ 195

13.3.1 Beispiel:Prüfmaschine $\ldots \ldots \ldots \ldots \ldots \ldots \ldots \ldots \ldots . \quad 196$

13.4 Entwurf von Reglern für robuste Regelqualität (RP) ........ 199

13.4.1 Main-Loop-Theorem .......................... 199

13.4.2 Analyse robuster Regelqualität $\ldots \ldots \ldots \ldots \ldots \ldots \ldots .200$

13.4.3 Berechnung von Reglern für RP ............... 202

13.4.4 Beispiel: Optimierung der robusten Regelqualität ..... 206

13.4.5 MATLAB-Befehle zum Beispiel 13.4.4

(Entwurf eines Regles für robuste Regelqualität) ..... 213

Literaturverzeichnis .................... 217

Mathematischer Anhang $\ldots \ldots \ldots \ldots \ldots \ldots \ldots \ldots \ldots .223$

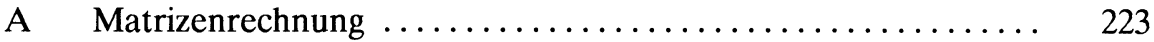

A.1 Rechenregeln für Transposition und Inversion ....... 223

A.1 Rechenregeln für Determinanten .............. 223

A.2 Matrix-Analysis ......................... 223

B Rechenregeln für Zustandsdarstellungen .............. 224

B.1 Basistransformation der Zustandsgrößen ........... 224

B.2 Inversion .............................. 224

B.3 Konjugiert komplexe Transposition .............. 224

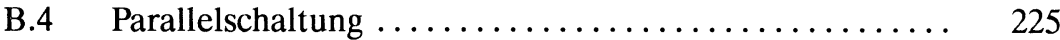

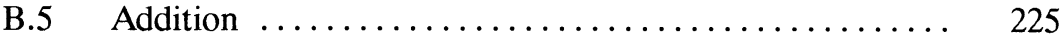

B.6 Multiplikation ........................... 225

B.7 Rückkopplung I $(\mathrm{D} 1=[0]) \ldots \ldots \ldots \ldots \ldots \ldots \ldots .226$

B.8 Rückkopplung II $(\mathrm{D} 1=[0]) \ldots \ldots \ldots \ldots \ldots \ldots \ldots, 226$

Sachverzeichnis ......................... 227 